\title{
Untersuchungen \\ über den Druck in den kleinsten Blutgefässen der menschlichen Haut.
}

\author{
II. M itteilu $\mathrm{g}$. \\ Ergebnisse der mit dem 0chrometer angestellten Versuche. - \\ Das Hautmanometer. - Vergleichende Untersuchungen mit beiden \\ Apparaten. \\ Von \\ Prof. Dr. Adolr Basler, \\ Assistent am physiologischen Institut in Tübingen.
}

(Mit 6 Textfiguren.)

Nachdem der Apparat zur Beurteilung des Blutdruckes in den Kapillaren, den ich Ochrometer genannt habe ${ }^{1}$ ), eine befriedigende Form erhalten hatte, ging ich dazu über, mit ihm an verschiedenen Personen - hauptsächlich Studenten - eine grosse Zahl von Bestimmungen auszufïhren. Dabei gelangte ich immer wieder zu dem auch in der ersten Mitteilung erwähnten Ergebnis, dass bei fast allen untersuchten Personen der geringste Druck, welcher ein Erblassen der dorsalen Haut der Fingerspitzen verursachte, im Mittel 90 bis $120 \mathrm{~mm}$ Wasser $=6,6-8,8 \mathrm{~mm}$ Quecksilber beträgt. Bei einigen Versuchspersonen wurden allerdings auch höhere Druckwerte gefunden.

Zum Zweck einer Vergleichung der gefundenen Werte mit früheren Angaben seien die Ergebnisse der verschiedenen Autoren in aller Kürze mitgeteilt.

Der erste, welcher den Druck in den kleinen Hautgefässen maass, war v. $\mathrm{Kries}^{2}$ ). Er stellte am Fingerrücken einen Druck von $513 \mathrm{~mm}$ Wasser $=37,7 \mathrm{~mm}$ Quecksilber fest.

1) A. Basıer, Untersuchungen über den Druck in den kleinsten Blutgefässen der menschlichen Haut. I. Mitt. Pflüger's Arch. Bd. 147 S. 393 (395). 1912.

2) N. v. Kries, Über den Druck in den Blutkapillaren der menschlichen Haut. Ber. d. Sächs. Gesellsch. d. Wiss. Bd. 27 S. 149 (151). 1875.

Pflüger's Archiv für Physiologie. Bd. 157. 
Natanson ${ }^{1}$, der den Kapillardruck bei Abschnürung des Armes untersuchte, fand den sehr hohen Wert $945 \mathrm{~mm}$ Wasser = $70,5 \mathrm{~mm}$ Quecksilber. Dabei wurde allerdings so stark gedrückt, bis die Stelle vollkommen weiss war.

Die Arbeit von $\mathrm{H}$. und B. Ballantine war mir leider nicht zugänglich, so dass es mir nicht möglich ist, 'ihre Ergebnisse mitzuteilen.

Rotermund ${ }^{3}$ ), dem ein grosses klinisches Material zur Verfügung stand, fand bei Männern einen Kapillardruck von $20-42 \mathrm{~mm}$ Quecksilber; beim weiblichen Geschlecht war er etwas niedriger.

Schille $r^{4}$ ), welcher vor der Beobachtung die Hand in Wasser tauchte, sah bei einer Wassertemperatur von $30-35^{\circ} \mathrm{C}$. die Haut erblassen, wenn der Fick'sche Ophthalmotonometer, der zum Konprimieren der Haut verwenclet wurde, eine Spannung von $15 \mathrm{~g}$ anzeigte. Da das Scheibchen des genannten Apparates, welches auf die Haut gesetzt wurde, $6-7 \mathrm{~mm}$ Durchmesser hat ${ }^{5}$ ), so entspricht einer Spannung von $15 \mathrm{~g}$ ein hydrostatischer Druck von etwa $40 \mathrm{~mm}$ Quecksilber.

Bei allen diesen Untersuchungen wurde die Haut mit einer Glasplatte gedrückt. Aber auch die zweite Reihe der vorliegenden Beobachtungen, bei denen über der zu untersuchenden Hautpartie der Luftdruck erhöht wurde, führte zu ähnlichen Ergebnissen.

Roy und $\mathrm{Brown}{ }^{6}$ ) konnten beobachten, dass in der Schwimmhaut des Frosehes bei einem von aussen einwirkenden Druck von $100-150 \mathrm{~mm}$ Wasser die Blutbewegung in den Kapillaren und Venen aufhörte. Damit die Strömung in den Arterien gehemmt wurde, war ein Druck von $200-350 \mathrm{~mm}$ Wasser nötig. Dabei ist allerdings nur die Rede von einem Aufhören der Bewegung, nicht

1) G. Natanson, Über das Verhalten des Blutdruckes in den Kapillaren nach Massenumschnürungen. Dissert. S. 14. Königsberg 1886.

2) H. and B. Ballantine, Journ. of Boston. Soc. of med. science vol. 3 p. 330 . 1899 .

3) H. Rotermund, Über den Kapillardruck, besonders bei Arteriosklerose. Inaug.-Dissert. S. 10. Marburg 1904.

4) W. Schiller, Über den Einfinss der Temperatur auf den Druck in den Kapillaren der Haut. Physiol. Zentralbl. Bd. 24 S. 391 (392). 1911.

5) A. Fick, Über Messung des Druckes im Auge. Pflüger's Arch. Bd. 42 S. 86. 1888. - Ges. Schriften Bd. 3 S. 456 (458). 1904.

6) Ch. Roy und J. G. Brown, Neue Methode, den Blutdruck in den kleinen Arterien, Venen und Kapillaren zu messen. Arch. f. (Anat. u.) Physiol. 1878 S. 158 (159). - The blood pressure and its variations in the arterioles, capillaries and smaller veins. Journ. of Physicl. vol. 2 p. 323. 1879/1880. 
Untersuchungen über den Druck in den kleinsten Blutgefässen etc. 347

von Entleerung der Gefässe. Aber sicher werden durch den Druck bestimmte Teile des Gefässsystems auch entleert, wodurch ein Erblassen der Haut im ganzen bedingt ist.

Natanson ${ }^{1}$ ) brachte erst durch einen Druck von $12-24 \mathrm{~mm}$ Quecksilber die Bewegung in den Kapillaren und Venen der Froschschwimmhaut zum Verschwinden.

Lapinsky ${ }^{2}$ ) fand sogar mit einer ähnlichen Methode bei kleineren Fröschen einen Kapillardruck von 200-600, bei grösseren einen solchen von $400-800 \mathrm{~mm}$ Wasser. Bei diesen Versuchen werden sicher nicht nur die Kapillaren, sondern auch schon ziemlich grosse Arterien zusammengepresst. Denn Hofmeister ${ }^{3}$ ) fand in der Art. cruralis der Kröte einen mittleren Druck von nur $44-45 \mathrm{~mm} \mathrm{Hg}=598,4-612 \mathrm{~mm}$ Wasser. Es ist aber sicher anzunehmen, dass dieser Wert bei Fröschen nicht grösser ist.

v. $\mathrm{Basch}^{4}$ ) ermittelte als minimale Werte des Kapillardruckes im Kaninchenohr 21-25 mm Quecksilber. An sich selbst fand er bei mehrmaliger Messung einen solchen von $28-30 \mathrm{~mm} \mathrm{Hg}^{5}$ ). Als normal betrachtet er einen Kapillardruck zwischen 25 und $30 \mathrm{~mm}$ Quecksilber ${ }^{6}$ ).

v. Recklinghausen ${ }^{7}$ ) fand - wenn ich seine Tabelle richtig verstehe - als Kapillardruck an den Fingerspitzen bei horizontal gehaltenem Arm den sehr hohen Wert von $75 \mathrm{~cm}$ Wasser, das sind $52,5 \mathrm{~mm}$ Quecksilber.

L o mbard ${ }^{8}$ ), der das Verschwinden der verschiedenen Blut-

1) G. Natanson, l. c. S. 16 .

2) M. Lapinsky, Studien über die lokale Blutzirkulation im Bereiche gelähmter Nerven. Arch. f. (Anat. u.) Physiol. 1899 Suppl. S. 477 (489).

3) F. Hofmeister, Beiträge zur Lehre vom Kreislauf der Kaltblüter. Pflüger's Arch. Bd. 44 S. 360 (367). 1889.

4) S. v. B a sch, Experimentelle und klin. Untersuchungen über den Kapillardruck. Intern. Beitr. zur inn. Med. Bd. 1 S. 65 (69). 1903.

5) S. v. Basch, 1. c. S. 74 .

6) S. v. Basch, Über die Messung des Kapillardruckes am Menschen und deren physiologische und klinische Bedeutung. Wiener klin. Rundschau Bd. 14 S. 549 (551). 1900.

7) H. v. Recklinghausen, Unblutige Blutdruckmessung. 3. Mitt. Arch. f. experim. Pathol. u. Pharmakol. Bd. 55 S. 463 (484). 1906.

8) W. P. Lombard, Der Blutdruck in den Kapillaren und kleinen Venen der menschlichen Haut. Zentralbl. f. Physiol. Bd. 25 S. 157. 1912. - The Blood Pressure in the Arterioles, Capillaries and small veins of the human Skin. Americ. Journ. of Physiol. vol. 29 p. 335. 1912. 
gefässe der Haut bei zunehmendem Druck beobachtete, stellte in dem subpapillären Venenplexus einen Druck von 10-15 mm Quecksilbe: fest, in den am leichtesten komprimierbaren Kapillaren einen solchen von $18-22 \mathrm{~mm} \mathrm{Hg}$.

Die bisher gefundenen Werte des Kapillardruckes seien zur bequemeren Orientierung in Form einer Tabelle zusammengestellt.

\begin{tabular}{|c|c|c|}
\hline \multirow{2}{*}{ Beobachter } & \multicolumn{2}{|c|}{ Kapillardruck in Millimetern } \\
\hline & Wasser & Quecksilber \\
\hline 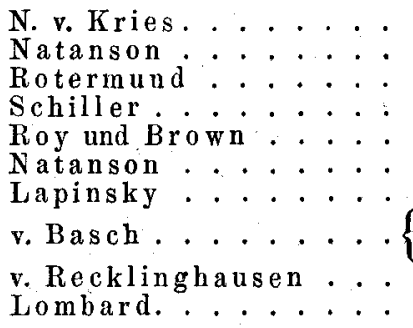 & $\begin{array}{c}513 \\
945 \\
272-571 \\
544 \text { (ungefähr) } \\
100-150 \\
163,2-326,4 \\
200-800 \\
285,6-340 \\
380,8-408 \\
750 \\
244,8-299,2\end{array}$ & $\begin{array}{c}37,7 \\
70,5 \\
20-42 \\
40 \text { (ungefähr) } \\
7,4-11,0 \\
12-24 \\
14,7-58,8\} \text { (Schwimmbaut } \\
21-25 \text { (Kaninchen) } \\
28-30 \text { (an sich) } \\
52,5 \\
18-22\end{array}$ \\
\hline
\end{tabular}

In jünster Zeit hat Herr Goldmann mit dem Ochrometer den Einfluss von verschiedenen Temperaturen auf den Kapillardruck untersucht und hat unter anderem gefunden, dass eine Wassertemperatur von ungefähr $25-30^{\circ} \mathrm{C}$. den normalerweise vorhandenen kleinsten Kapillardruck nicht ändert. Diese Tatsache hängt damit zusammen, dass Wasser von $25-30^{\circ} \mathrm{C}$. der Haut ungefähr ebensoviel Wärme entzieht wie sonst die den Finger ungebende Luft. Erhöhte man indessen die Temperatur des Wassers, dann wurde der Kapillardruck mit zunehmender Temperatur immer grösser, eine Erscheinung, die durch Erweiterung der kleinsten Arterien bei Anwendung von Wärme zur Genüge erk]ärt sein dürfte. Goldmann fand ausserdem, dass auch nach Abkühlung der untersuchten Stelle ein grösserer Druck angewendet werden musste, um ein Erblassen der Haut wahrnehmen zu können, doch soll auf diese sowie die anderen von ihm beobachteten Tatsachen hier nicht näher eingegangen werden, da sie in der Publikation von Goldmann genau erörtert werden. Es sei an dieser Stelle nur auf den einen Punkt hingewiesen, dass auch Goldmann als normalen Kapillardruck stets einen solchen von $80-100 \mathrm{~mm}$ Wasser fand. 
Untersuchungen über den Druck in den kleinsten Blutgefässen etc. 349

Landerer ${ }^{1}$ konnte mit dem Ochrometer bei verschiedenen Krankheiten ein abweichendes Verhalten des Kapillardruckes gegenüber der Norm feststellen.

Dabei hat aber Landerer ${ }^{2}$ ) im Gegensatz zu meinen eigenen und den unter meiner Leitung angestellten Untersuchungen stets einen höheren Kapillardruck gefunden, bei gesunden Männern 17 bis $25 \mathrm{~mm}$ Quecksilber. Seine Ergebnisse stehen also zwischen denen der früheren Forseher und meinen eigenen. Zur Erklärung dieses Unterschiedes sei daran erinnert, dass die Gefässe der Haut die verschiedensten Grössen aufweisen. Die grössten Gefässe der arteriellen Seite sind mit Blut gefült, das unter höherem Druck steht als der Inhalt der kleinsten kapillären Verzweigungen und in letzteren ist der Druck wieder grösser als in den weiten Gefässen der venösen Seite. Wenn ganz allgemein vom Kapillardruck gesprochen wird, so werden unter diesem Begriff alle die verschiedenen Werte subsummiert. Dabei handelt es sich eben nicht ausschliesslich um Kapillaren im anatomischen Sinn, sondern auch um kleinste Arterien und kleinste Venen, was man auch aus den Untersuchungen von Lombard ersieht.

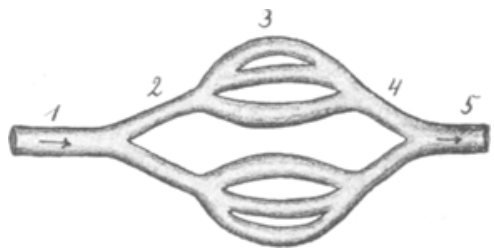

Fig. 1. Schematische Zeichnung der Gefässverzweigungen. 1 und 2 arterielle Gefässe, 3 Kapillaren, 4 und 5 venöse Gefässe.

In Fig. 1 sei die Blutgefässverteilung eines kleinen Hautbezirkes schematisch dargestellt. Dabei wurde jedoch, um die Skizze möglichst übersichtlich zu machen, die anatomische Anordnung der Gefässe in der menschlichen Haut im einzelnen nicht berücksichtigt. Die Zeichnung hält sich eher an den Verlauf der Blutbahnen in der Froschpfote. Mit 1 sind arterielle Gefässe einer bestimmten Grösse bezeichnet, mit 2 die daraus entspringenden kleineren. Diese verzweigen sich wieder, wodurch Kapillarschlingen zustande kommen, die als 3 bezeichnet sein mögen. Sie sollen sich vereinigen zu einem venösen Gefässchen 4, welches mit noch einem anderen sein Blut in die Vene 5 ergiesst.

Wenn man mit dem Druck, der von aussen einwirkt, allmählich steigt, dann werden zuerst diejenigen Gefässe komprimiert, in denen

1) R. Landerer, Zur Frage des Kapillardruckes. Zeitschr. f. klin. Med. Bd. 78 H. 1 u. 2. 1913.

2) R. Landerer, l. c. Separatdruck S. 3. 
der kleinste Druck herrscht, das sind die in der Skizze mit 5 bezeichneten. Ihr Zusammenfallen bedingt die erste Farbenveränderung. Bei weiterer Zunahme des Druckes folgen der Reihe nach die Äste $4,3,2,1$, wobei jedesmal, wenn eine neue Kategorie von Gefässen verschlossen wird, eine hellere Tönung der Hautfarbe entsteht. Es ist deshalb nötig, bei Untersuchungen mit dem Ochrometer immer den gleichen, am besten den allerkleinsten Farbenumschlag herzustellen.

Land erer hat aus irgendeinem Grunde - vielleicht wegen unzureichender Beleuchtung - den allerersten Farbenumschlag nicht bemerkt, sondern erst einen stärkeren, und so hat er vielleicht den Druck in den Kapillaren 4 oder 3 gemessen, während ich den Druck in den Gefässen 5 ermittelt habe. An und für sich sind die Ergebnisse von Landerer natülich deshalb nicht weniger richtig; sie beziehen sich nur auf eine andere Kategorie von Gefässen.

Man sieht aber hieraus, dass streng genommen nur die Bestimmungen von einem und demselben Beobachter unter sich verglichen werden dürfen. Deshalb schien es mir wünschenswert, eine Methode ausfindig zu machen, welche objektiv fest bestimmbare Ergebnisse liefert, mit denen sich dann die mit dem Ochrometer gefundenen Werte gewissermaassen eichen lassen. Das dringende Bedürfnis für ein Kontrolverfahren ergibt sich auch noch aus einem anderen Grunde.

Bei der Konstruktion des Ochrometers war ich zwar bestrebt, die Bedingungen für eine genaue Ablesung möglichst günstig zu gestalten, und die mit dem Apparat ausgeführten Versuche haben auch gezeigt, dass die einzelnen Bestimmungen gut miteinander übereinstimmen. Aber es ist nicht $\mathrm{zu}$ vergessen, dass ich mich im wesentlichen des schon von v. Kries angewendeten Prinzips bediente, bei dem der Druck in den Kapillaren doch nur indirekt festgestellt werden kann. Die Methode ist also etwa zu vergleichen mit dem von Riva-Rocei $\left.{ }^{1}\right)$ angegebenen, und namentlich von v. Reckling hausen ${ }^{2}$ ) verbesserten Verfahren zur Bestimmung des arteriellen

1) Riva-Rocei, Un nuovo Sphigmomanometro. Torino 1896. Zitiert nach Müller und Blauel. Deutsches Arch. f. klin. Med. Bd. 21 S. 517. 1907.

2) H. v. Recklinghausen, Unblutige Blutdruckmessung. II. Abhandl. Arch. f. experim. Pathol. u. Pharmakol. Bd. 55 S. 412. 1906. 
Blutdruckes beim Menschen. In beiden Fällen handelt es sich darum, das Blutgefäss unter einen solchen Druck zu setzen, dass es eben zusammengepresst wird, wobei man von der Annahme ausgeht, dass diese Formveränderung eintritt, sobald der äussere Druck um eine Kleinigkeit grösser geworden ist als der, unter dem das Blut steht. Seither wurden die verschiedenen Modifikationen dieser unblutigen Blutdruckmessung einer teils theoretischen, teils experimentellen Nachprüfung unterworfen ${ }^{1}$ ).

Von solchen Untersuchungen sei nur die von Müller und $\mathrm{Blauel}{ }^{2}$ ) erwähnt. In eine Arterie des menschlichen Unterarmes wurde eine Kanüle eingeführt und diese mit einem Manometer verbunden, das die pulsatorischen Druckschwankungen registrierte. Dadurch war man über den arteriellen Blutdruck in jedem Augenblick zuverlässig unterrichtet. Jetzt wurde der Apparat von RivaRocci in Tätigkeit gesetzt und festgestellt, bei welchem Druck der Armmanschette die pulsatorischen Blutdruckschwankungen verschwanden. Bei diesen Untersuchungen zeigte sich, dass die unblutige Druckmessung keine Werte gab, die dem wirklichen Blutdruck genau entsprachen. Doch stimmten die Ergebnisse mit dem wirklichen Druck am besten überein bei Anwendung der von Recklinghausen angegebenen breiten Manschette.

Diese Erfahrungen lassen sich aber nicht auf die Kapillaren anwenden, denn wir haben es dabei mit ganz anderen physikalischen Bedingungen zu tun. So ist z. B. bei den Kapillaren das Einzellumen versch windend klein im Vergleich zu den umgebenden Geweben.

Schon derartige Erwägungen machen es wünschenswert, den in den Kapillaren herrschenden Druck direkt manometrisch zu bestimmen und die Ergebnisse mit den mit dem Ochrometer gefundenen Werten zu vergleichen.

\section{Grundgedanken einer objektiven Methode.}

Bringt man an der Fingerbeere eine kleine Stichverletzung an, dann entströmt der Wunde ein Tropfen Blut, der aus einer grösseren

1) Vgl. F. Nicolai, Die Mechanik des Kreislaufes. W. Nagel's Handb. d. Physiol. Bd. 1 S. 661 (707). Braunschweig 1909. - O. Frank, Hämodynamik. R. Tigerstedt's Handb. d. physiol. Meth. Bd.2 Abt. 4 S. 1 (216). Leipzig 1911.

2) O. Müller und K. Blauel,'Zur Kritik des Riva-Rocci'schen und Gärtner'schen Sphygmomanometers. Deutsches Arch. f. klin. Med. Bd. 21 S. 517. 1907. 
Zahl kleiner in der Haut liegender Gefässe stammt, welche bei dem Einstich eröffnet wurden. Um was für Gefässe es sich dabei handelt; darauf soll später näher eingegangen werden. Ich stellte mir nun die Aufgabe, den Druck zu messen, unter dem das Blut aus dem Stichkanal ausfliesst, und konstruierte zu diesem $Z$ wecke einen kleinen Apparat, der am zweckmässigsten als $\mathrm{Hautmanometer} \mathrm{be-}$ zeichnet wird.

Das prinzipiell Neue an dieser Methode ist, dass die Kapillaren dabei eröffnet werden, während man sich bisher damit begnügte, sie von aussen her zusammenzupressen.

\section{Besehreibung des Hautmanometers.}

Den von mir verwendeten Apparat, der von Herrn Universitätsmechaniker Albrecht hergestellt wurde, habe ich vor etwa einem halben Jahr in aller Kürze beschrieben ${ }^{1}$ ). Er besteht aus einem

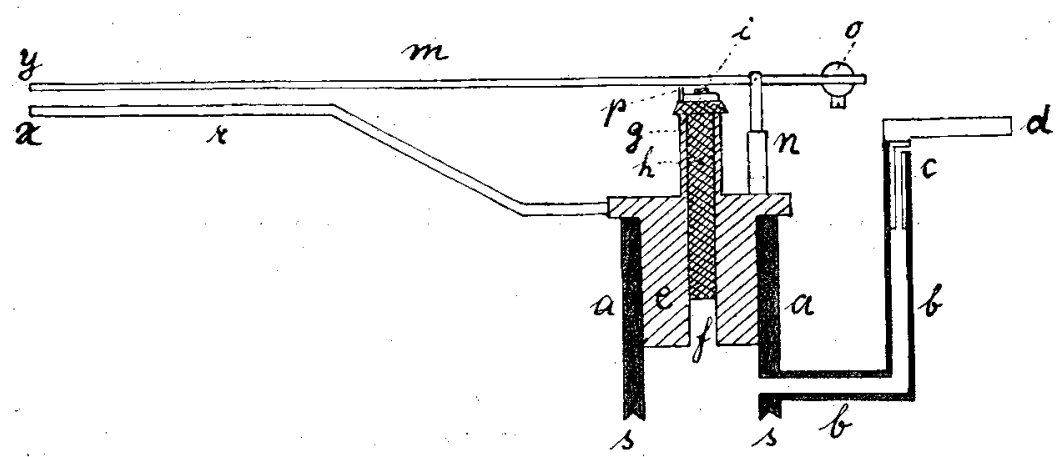

Fig. 2. Durchschnitt des Hautmanometers (etwas vergrössert). $m=$ Hebel, $\gamma=$ Stäbchen zur Beurteilung seines Ausschlages, $p=$ Aluminiumplättchen als Unterlage für den Hebel, $h=$ Koiben aus Hartgummi, $i=$ Gummifaden (quer getroffen), $f=$ Kolbenzylinder, $b=$ Röhrchen, durch das die überschüssige Flüssigkeit entweichen kann.

$14 \mathrm{~mm}$ hohen Rohrstück $a$ (Fig. 2) von $8 \mathrm{~mm}$ Lichtweite, das mit seiner unteren Öffnung wasserdicht auf der Haut befestigt wird. Wie das geschieht, wird weiter unten beschrieben. In dasselbe ist ein ungefähr $1 \mathrm{~mm}$ weites Röhrchen $b$ eingescbraubt, das in $9 \mathrm{~mm} \mathrm{Ab}$ stand abgeknickt und nach aufwärts gebogen ist. Am oberen Ende

1) A. Basler, Demonstration eines Apparates zur Untersuchung des Druckes. in đen Blutkapillaren der Haut. Münch. med. Wochenschr. Bd. 60 S. 1972. 1913. 
besitzt es einen hahnartigen Verschluss $c$ mit einem kleinen Griff $d$. Steht der Griff $d$ so, wie er in Fig. 2 skizziert ist, dann kommuniziert der Innenraum des Röhrehens $b$ durch eine enge Öffnung mit aussen. Soll jedoch die Verbindung aufhören, dann braucht man nur den Griff $d$ etwas nach der Seite zu drehen. In das Rohrstück $a$ passt genau ein zylindrischer Zapfen aus Messing $e$ (einfach schraffiert), der in seiner Achse von einem $2 \mathrm{~mm}$ weiten senkrechten Kanal $f$ durchsetzt wird. Zur Verlängerung des Kanals $f$ wurde auf das Stück $e$ ein Hohlzylinder $g$ aufgesetzt. So entstand ein zylindrischer Hohlraum von $16 \mathrm{~mm}$ Länge. Darin bewegt sich ein gut passender Kolben aus Hartgummi $h$ (gekreuzt schraffiert), der natürlich vor dem Gebrauch gut geölt werden muss. (Am besten eignet sich dazu

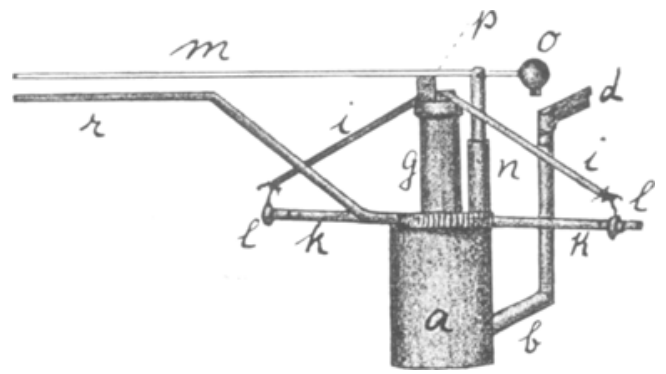

Fig. 3. Perspektivische Ansicht des Hautmanometers. $i=$ Gummifaden, $k k=$ Stäbchen zu seiner Befestigung, $m=$ Hebel, $r=$ Stäbchen zur Beurteilung seiner Bewegung, $p=$ Aluminiumplättchen als Unterlage tür den Hebel.

feines Maschinenöl.) Auf die obere Fläche des Kolbens wird eine Zugwirkung nach unten ausgeübt durch den Gummifaden $i$ (vgl. Fig. 3), der an zwei Stäbchen $k k$ durch je eine kleine verschiebliche, mit einem Häkchen versehene Hülse $l$ befestigt ist. Auf dem Durchschnitt Fig. 2 ist der Gummifaden natürlich nur im Querschnitt zu sehen.

Denkt man sich nun die untere Öffnung des Robres $a$ verschlossen und das ganze Hohlsystem mit Flüssigkeit gefüllt, dann muss, wenn eine Volumenzunahme der Flüssigkeit stattfindet, die mit einem gewissen Druck vor sich geht, der Kolben so lange steigen, bis der Gummifaden eine Spannung erreicht bat, die dem Druck der Flüssigkeit im Innern des Apparates gerade das Gleichgewicht hält. Die Bewegungen des Kolbens werden durch den Hebel $m$ zebnmal vergrössert. Dieser Hebel aus Aluminium dreht sich sehr leicht um 
eine Achse, die an dem in seiner Höhe verstellbaren Aluminiumstäbchen $n$ angebracht ist. Zum Äquilibrieren des ganzen Hebels dient eine Kugel o, welche mit einem kleinen Schräubchen befestigt wird. Die Kugel wird so gestellt, dass der lange Hebelarm mit einem Übergewicht von nur einigen Milligramm auf seine Unteriage drückt. Er wird in horizontaler Stellung gehalten dadurch, dass er auf einem an der Oberfläche des Kolbens $h$ befestigten Aluminiumstreifen $p$ aufliegt. Da die kleinen Einrichtungen, die an der oberen Fläche des Kolbens angebracht sind, auf den Fig. 2 und 3 schwer zu erkennen sind, sei die obere Kolbenhälfte in Fig. 4 nochmals, und $z$ war in stark vergrössertem Maassstabe dargestellt.
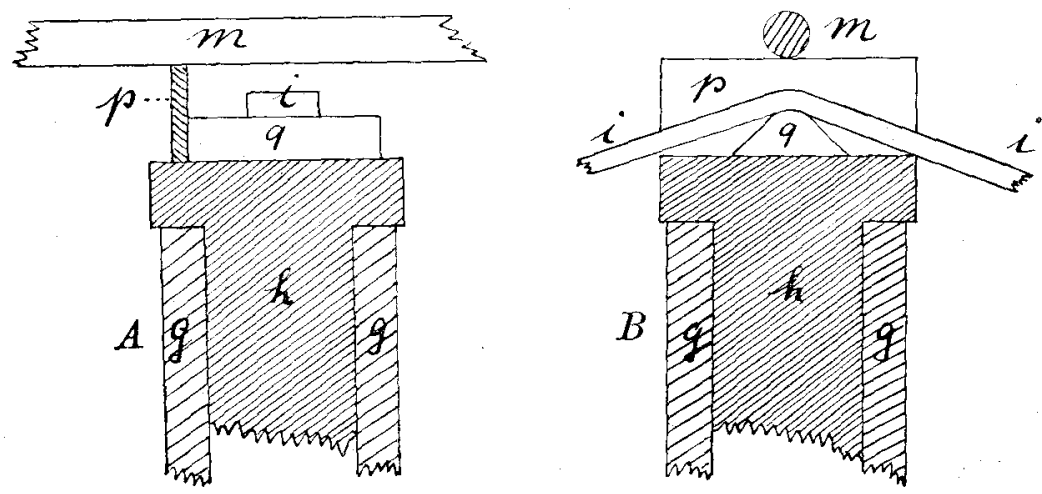

Fig. 4. Oberes Kolbenende mit den anstossenden Teilen des Apparates. $A$ von der Seite, $B$ von vorn gesehen, $h=$ Kolben, $g=$ Kolbenzylinder, $p=$ Unterlage für den Hebel, $m=$ Hebel, $q=$ Unterlage für den Gummifaden, $i=$ Gummifaden.

Um die Einstellungen der Spitze des Hebels $m$ richtig beurteilen $\mathrm{zu}$ können, ist an dem oberen Rand des Zapfens $e$ (Fig. 2) ein Stäbchen eingeschraubt, welches so gebogen ist, dass sich sein vorderes Ende $x$ nahe bei der Hebelspitze $y$ befindet. Dieses Stäbchen ist auf Fig. 2 und 3 mit $r$ bezeichnet. Die Entfernung der Punkte $x$ und $y$, die mit einem Zirkel bestimmt wird, gibt die Grösse des Hebelausschlags an.

Bei der Herstellung des beschriebenen Manometers waren hauptsächlich zwei Bedingungen maassgebend, denen er entsprechen sollte. Es mussten fürs erste sehr geringe Flüssigkeitsmengen ausreichen, um einen messbaren Ausschlag zu verursachen, und ausserdem wurde verlangt, dass der Apparat sehr kleine Druckwerte anzeigt. Das ist der Grund, weshalb ihm gerade die Form des Kolbenmanometers 
gegeben wurde. Dass er der zuerst genannten Anforderung entspricht, geht aus einer Betrachtung der Maasse hervor.

Der Durchmesser des Kolbenzylinders $f$ (Fig. 2, S. 352) misst $2 \mathrm{~mm}$, also sein Querschnitt $1^{2} \pi=3,14 \mathrm{qmm}$. Bei einer Verschiebung des Kolbens um einen Millimeter muss demnach der Flüssigkeitszuwachs in dem ganzen Apparat 3,14 cbmm betragen. Wenn sich also der Kolben bei einem Versuch um $3 \mathrm{~mm}$ heben würde (eine so grosse Verschiebung kommt nie vor), wären noch nicht einmal $10 \mathrm{cbmm}$ Blut notwendig. So viel Blut hat man aber schon bei verhältnismässig kleinen Eingriffen zur Verfügung. Ich erinnere z. B. nur daran, dass man bei vielen Methoden der Hämoglobinuntersuchung $20 \mathrm{cbmm}$ Blut gebraucht und auch erhält.

Der zweiten Anforderung, wurde dadurch entsprochen, dass dem auf den Kolben von unten her wirkenden Blutdruck durch einen recht dünnen Gummifaden entgegengewirkt wird.

Nach meinen mit dem Ochrometer gewonnenen Ergebnissen war anzunehmen, dass Drücke bestimmt werden müssen, die zwischen 50 und $200 \mathrm{~mm}$ Wasser liegen. Daraus lässt sich berechnen, wie gross die elastische Kraft des Gummifadens sein muss, die auf das obere Kolbenende wirkt. Eine $1 \mathrm{~cm}$ hohe Wassersäule hat die Masse $1 \mathrm{~g}$, wenn die Bodenfläche $100 \mathrm{gmm}$ gross ist. Bei einer Bodenfläche von $1 \mathrm{qmm}$ beträgt die Masse der Wassersäule von $1 \mathrm{~cm}$ Höhe $0,01 \mathrm{~g}$. Auf dem Zylinderquerschnitt, der 3,14 qmm gross ist, und somit auf der unteren Fläche des Kolbens, lastet demnach eine $1 \mathrm{~cm}$ hohe Wassersäule mit einem Gewicht von $0,0314 \mathrm{~g}$. Bei $\zeta \mathrm{cm}$ Wassersäule beträgt die Belastung $5 \cdot 0,0314 \mathrm{~g}=0,157 \mathrm{~g}$ und bei $20 \mathrm{~cm}$ Wasserhöhe $20 \cdot 0,0314 \mathrm{~g}=0,628 \mathrm{~g}$. Man gebraucht also zur Erzeugung des Gegendruckes sehr dünne Gummifäden, nämlich solche, die sich bei einer Belastung mit $0,1-0,6 \mathrm{~g}$ schon merklich ausdehnen. Zur Herstellung derselben benutzte ich Fingerlinge aus dünnem Gummi, wie sie im Handel sind. Aus ihnen schnitt ich ungefähr $1 \mathrm{~cm}$ breite Streifen, die mit Stärkekleister zwischen je zwei Papierblätter geklebt wurden. Nach dem Trocknen lässt sich eine solche Platte leicht in Streifen von $1 / 3-1 \mathrm{~mm}$ Breite schneiden. Werden diese Streifen in Wasser gelegt, dann löst sich die an beiden Seiten aufgeklebte Papierschicht ab, und man hat jetzt die Gummifäden zum Gebrauch fertig. Sie werden an beiden Enden mit Fadenschlingen versehen zum Einhängen in die Häkchen des Apparates. 
Für meine Zwecke musste das Manometer zwar geringe Druckwerte anzeigen, aber es brauchte sich nicht schnell einzustellen, denn das Kapillarblut fliesst ohnedies recht langsam aus der Wunde, und Schwankungen des Druckes kommen nicht in Betracht. Aus diesem Grunde brauchte auf Eigenschwingungen des Apparates und auf deren

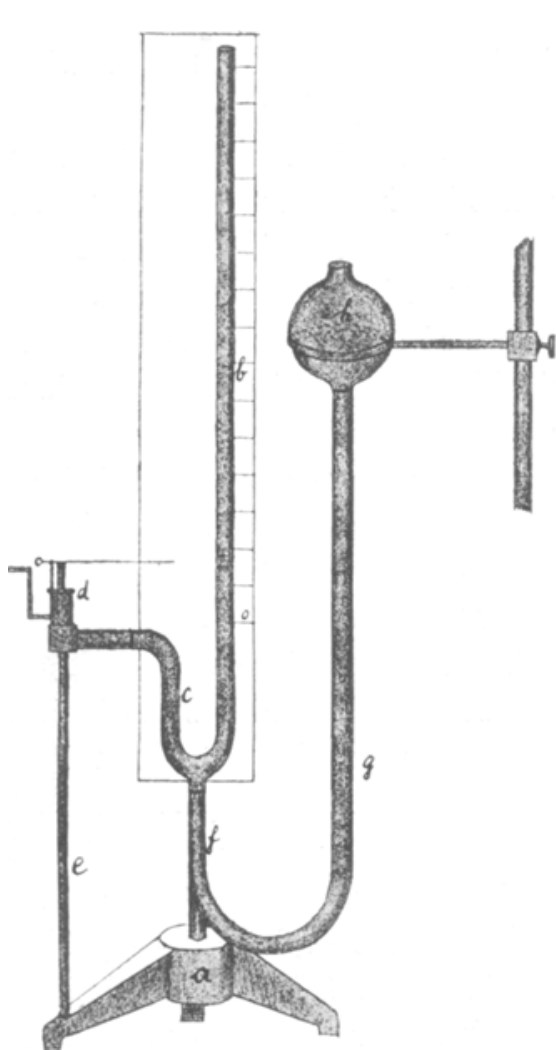

Fig. 5. Aufstellung zum Kalibrieren des Apparates. $b c=\mathrm{U}$-förmig gebogene Glasröhre, $h=$ mit Wasser gefüllte Kugel zur Herstellung des Druckes, $d=$ Hautmanometer.

Dauer bei der Konstruktion keine Rücksicht genommen zu werden. Sollte von dem Apparat verlangt werden, Druckschwankungen getreu anzuzeigen, dann müsste er eine wesentlich andere Form erhalten ${ }^{1}$ ).

\section{Kalibrierung des Mano- meters.}

Um zu wissen, welchem Druck ein bestimmter Ausschlag des Hebels entspricht, ist es nötig, den Apparat auszuwerten. Dazu habe ich folgende Einrichtung verwendet. Auf einem Dreifuss $a$ (Fig. 5) ist eine auf einem Brettchen montierte, u-förmig gebogene Glasröhre mit einem langen Schenkel $b$ und einem kurzen $c$ befestigt.

Der Schenkel $c$ ist durch einen kurzen Gummischlauch mit einer Metallkapsel verbunden, deren obere Öffnung so gross ist, dass das Messingrohr a des Manometers (vgl. Fig. 2 und 3, S. 352 und 353) gerade hineinpasst und in ihr wasserdicht hält. Die Kapsel wird getragen durch einen senkrechten, von dem einen Fuss des Statives aufsteigenden Stab e. An der Umbiegungsstelle des Glas-

1) Vgl, O. Frank, Therorie des Kolbenmanometers. Zeitschr. f. Biol. Bd. 45 (N. F. Bd. 27) S. 464.1904. 
rohres $b c$ ist ein kurzes Rohrstück $f$ eingeschmolzen, das durch einen Gummischlauch $g$ mit der Glaskugel $h$ in Verbindung steht. Das ganze System wird mit Wasser gefüllt, dessen Druck sich natürlich je nach der Höhe der Glaskugel ändert. Der Druck lässt sich an einer neben dem Rohr $b$ angebrachten Skala ablesen. Um die Brauchbarkeit des Manometers und etwa demselben anhaftende Fehler zu erkennen, habe ich die Ausschläge des Apparates bei Armierung mit verschieden starken Gummifäden unter Anwendung der in Betracht kommenden Drücke untersucht.

So fand ich z. B. für einen bestimmten Gummifaden Werte, wie sie aus folgender Tabelle ersichtlich sind.

$\begin{array}{cc}\begin{array}{c}\text { Ausschlag der Hebelspitze } \\ \text { in Millimetern }\end{array} & \begin{array}{c}\text { Wasserdruck } \\ \text { in Millimeter }\end{array} \\ 1,5 & 0 \\ 1,8 & 40 \\ 5,0 & 60 \\ 5,5 & 70 \\ 10,0 & 90 \\ 12,0 & 100 \\ 16,0 & 110 \\ 18,0 & 120 \\ 20,0 & 130\end{array}$

Bei einer etwas stärkeren Spannung des Gummifadens ergaben sich die Werte:

$\begin{array}{cc}\begin{array}{c}\text { Einstellung der Hebelspitze } \\ \text { in Millimetern }\end{array} & \begin{array}{c}\text { Wasserdruck } \\ \text { in Millimetern }\end{array} \\ 1,0 & 0 \\ 2,0 & 20 \\ 4,0 & 50 \\ 7,5 & 90 \\ 8,0 & 100 \\ 9,5 & 120\end{array}$

Man muss aber berücksichtigen, dass die Elastizität des Gummifadens sich leicht ändert, und dass deshalb der Apparat von einem Versuch zum nächsten andere Werte geben kann. Deshalb habe ich sofort nach jeder Bestimmung mit Hilfe des in Fig. 5 dargestellten Apparates den zu dem beobachteten Hebelausschlag gehörigen Druck ermittelt. 


\section{Aufkleben des Apparates.}

Die grösste Schwierigkeit bereitete das Aufkleben des Apparates auf die zu untersuchende Hautstelle. Denn das Manometer soll nicht nur der Haut wasserdicht aufsitzen, sondern die Verbindung muss auch einen gewissen Druck aushalten. Ausserdem soll das Klebmittel die normale Beschaffenheit der Haut möglichst wenig ändern; vor allem soll es die Epidermis nicht zusammenziehen wie etwa Kollodium. Um ein möglichst gutes Verfahren ausfindig zu machen, wurden alle erdenkbaren Mittel probiert, jedoch mit wenigen Ausnahmen wieder verworfen. v. Basch, welcher einen Trichter aus Glas mit der engen Öffnung auf die Haut klebte und in dem Hohlraum die Luft komprimierte, verwendete Fischleim. Auch ich benützte diese Substanz, doch konnte ich mich bald davon überzeugen, dass sie für meinen Apparat unbrauchbar war, weil ein Durchsickern der Flüssigkeit nicht vermieden werden konnte.

Besser bewährte sich sogenanntes deutsches Heftpflaster. Das bej erhöhter Temperatur verfüssigte Heftpflaster wurde auf den unteren Rand des ebenfalls erwärmten Rohrstückes $a$ (Fig. 2) aufgestrichen und hierauf der Apparat im warmen Zustand auf die Haut aufgesetzt. Das Verfahren ist aber etwas umständlich, und die Versuche schienen mir nicht ganz einwandfrei, weil die Hautstelle, deren Kapillardruck bestimmt werden soll, vorher nicht unbedeutend erwärmt wird. Zudem konnte man sich nicht einmal sicher darauf verlassen, dass das Manometer ganz dicht aufsáss.

Ich verliess also auch dieses Mittel und benutzte Le ukoplastmasse, die mir in liebenswürdiger Weise von der Firma Beiers$\mathrm{dorf} \& \mathrm{Co}$. in Hamburg zur Verfügung gestellt wurde. Dieser Klebstoff, der von mir lange Zeit zur Verwendung kam, hat den Nachteil, dass er ziemlich lange zum Trocknen braucht.

Als sehr empfehlenswert erwies sich Klebwachs; damit es am unteren Rand des Röhrchens a (Fig. 2, S. 352) besser haftete, wurde in dasselbe eine kleine Rinne $s$ (Fig. 2) eingedreht. Das Klebwachs muss in ziemlich dicker Schicht aufgetragen werden. Bei mir und auch bei verschiedenen Studenten hielt der Apparat sowohl mit Leukoplastmasse wie mit Klebwachs ausgezeichnet. Aber bei starker Schwielenbildung, wie sie häuflg an den Händen von Arbeitern vorkommt, versagten beide Substanzen. Der Apparat fiel entweder sofort ab, oder aber während dem Versuche drang Flüssigkeit heraus. 
Um deshalb auch bei den Leuten aus dem Arbeiterstande, die bei dem zur Verfügung stehenden klinischen Material in der Regel die Hauptmasse bilden, Bestimmungen ausführen $\mathrm{zu}$ können, musste noch eine andere Art des Aufklebens ersonnen werden. $\mathrm{Zu}$ diesem Zwecke wurde das Rohr $a$ des Apparates in einen dickwandigen Gummischlauch gesteckt, in den nur ein Loch geschnitten war, durch welches das Röhrchen $b$ heraustrat. Der Gummiüberzug überragte den unteren Rand des Apparates, wie aus beistehender Skizze ersichtlich ist, um ungefähr $1 / 2-1 \mathrm{~mm}$. Fr ist in der Fig. 6 mit $t$ bezeichnet. Der Gummischlauch lässt sich nun leicht und ziemlich sicher mit Kanadabalsam festkleben. Der auf diese Weise befestigte Apparat hielt auch in den Fällen, wo alle bisher beschriebenen Mittel versagten. Ich würde deshalb diese Methode als den übrigen weit überlegen empfehlen, wenn ich nicht gleichzeitig ein Verfahren gefunlen hätte, das noch bessere Dienste tut, bei dessen Anwendung der Apparat nach dem Versuche nur mit Mühe von der Haut zu trennen ist. Stellt man nämlich den vorragenden Gummirand des

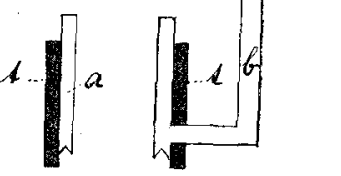

Fig. 6. Der Durchschnitt des Gummischlauches, in welchem der Apparat steckt, ist mit $t$ bezeichnet.

Apparates auf eine erhitzte Metallplatte, dann schmilzt die äusserste Gummischicht zu einer schmierigen, klebrigen Masse. Wird jetzt der Zylinder auf die Haut gesetzt, dann hält er so fest und absolut dicht wie mit keinem anderen Klebmittel.

\section{Einstich in die Haut.}

Nachdem der Teil $a$ des Manometers auf der Haut befestigt ist, müssen die kleinen Gefässe angeschnitten werden. Dies geschieht am einfachsten mit Hilfe der Francke'schen Nadel ${ }^{1}$ ). Dabei muss natürlich vermieden werden, dass man zu tief einsticht, weil man dabei schon richtige Arterien eröffnen und infolgedessen einen zu hohen Druck bestimmen könnte. Andererseits soll aber eine möglichst grosse Zahl von Gefässen verletzt werden, damit die austretende Blutmenge nicht zu klein ist. Deshalb habe ich die Klinge des

1) Die Beschreibung derselben bei K. Bürker, Gewinnung, qualitative und quantitative Bestimmung des Hámoglobins. Tigerstedt's Handb. d. physiol. Meth. Bd. 2, I S. 68 (76). Leipzig 1911. 
Apparates; wie es auch B ïrker empfiehlt, breit geschliffen. Dadurch entsteht eine Schnittwunde von ungefähr $1 \mathrm{~mm}$ Länge, aus der im allgemeinen genügend Blut für die Bestimmung austritt.

In der beschriebenen Weise wurde nach dem Aufsetzen des Manometers eine kleine Schnittwunde beigebracht; in einigen Fällen, wo die Blutung zu langsam erfolgte, deren zwei, manchmal sogar drei. Die Ausdehnung der Verletzung ändert natürlich nichts an dem Druck, unter dem das Blut ausströmt. Aus einer breiten Wunde tritt das Blut nur schneller aus als aus einer kleinen, und infolgedessen stellt sich das Manometer im ersten Falle schneller ein.

\section{Füllen des Apparates und Ausführung der Bestimmung.}

Nach eingetretener Blutung wurde der Teil $a$ des Manometers mit Hilfe einer Pipette mit $0,9 \%$ iger, auf $30^{\circ} \mathrm{C}$. erwärmter Kochsalzlösung angefüllt. Um die Gerinnung möglichst zu verhüten, wurde vorher etwas Hirudin darin gelöst. Nachdem sich jedoch herausgestellt hatte, dass die ganze Bestimmung in kürzester Zeit erfolgen kanu, liess ich späterhin das Hirudin weg, ohne dass irgendeine nachteilige Folge zu beobachten war. Nach Abnehmen des Manometers blutete die Wunde jedenfalls noch. Nur bei einigen Versuchen, in denen unvorhergesehene Zwischenfälle eintraten, wo z. B. der Apparat nicht dicht aufsass und frisch angeklebt werden musste, wodurch sich die Bestimmung verzögerte, trat mitunter Gerinnung ein. Aber es handelte sich dabei um Versuche, die an und für sich ausgeschieden werden mussten. Zur Verhütung der Gerinnung benützte ich lange Zeit hindurch zum Füllen des Apparates auch gesättigte Bittersalzlösung.

Auf das mit Flüssigkeit bis oben gefüllte Rohrstück $a$ wurde jetzt der übrige den Kolben tragende Teil des Apparates aufgesetzt. Der Zapfen $e$ (Fig. 2, S. 352) muss natürlich vorher eingefettet werden, damit er wasserdicht schliesst. Die Flüssigkeit wird dadurch zum grossen Teil verdiangt und entweicht durch die Öffnung bei $c$. Hierauf wird der Griff $d$ umgedreht, und sofort wird durch das aus der Schnittwunde strömende. Blut der Kolben langsam gehoben. Ich habe bei meinen Versuchen stets zunächst das hintere Ende des Hebels (bei der Kugel $o$, Fig. 2, S. 352) mit einem kleinen Reiter beschwert. Dadurch steht der lange Hebelarm nach oben, so dass während des Ansteigens des Kolbens keine Berührung zwischen dem am Kolben befestigten Plättchen $p$ und dem Hebel $m$ stattfindet. 
So ist die Reibung am Hebel vollkommen vermieden. Erst wenn der Kolben sich nicht mehr bewegte, wurde der Reiter beseitigt und der Hebel langsam heruntergelassen.

\section{Welche Gefïsse werden beim Stich verletzt?}

In der äusseren Haut sind die Gefässe in verschiedenen Schichten angeordnet. Die kleinen Arterien treten durch das subkutane Gewebe herein und bilden in der untersten Schicht des Coriums ein Geflecht, das kutane Netz. Von hier aus steigen Äste nach aufwärts, welche sich in einer oberflächlichen Lage des Coriums zu dem subpapillären Netz verschlingen. Dieses sendet $Z$ weige längs der Papillenreihen, aus denen die Kapillarschlingen der Cutispapillen entspringen. Das aus den Papillen zurückströmende Blut sammelt sich in venösen Ästchen, die zunächst, durch zahlreiche Anastomosen verbunden, den subpapillären Venenplexus darstellen. Von hier aus gelangt das Blut in ein dicht darunterliegendes zweites Netz; in einer tieferen Schicht des Coriums liegt ein drittes Netz, und schliesslich bilden die Venen in der Grenzschicht zwischen Corium und Subkutis nochmals ein viertes Netz, das etwa dem kutanen arteriellen Geflecht entspricht 1 ).

Alle diese Gefässe können nun bei dem Einstich in die Haut verletzt werden, vorausgesetzt, dass die Schneide die ganze Dicke der Cutis durchschnitten hat. Wird etwas weniger tief eingeschnitten, dann bleibt wohl das cutane Netz verschont. Aber auch in diesem Falle wird sicher eine ganze Menge kleinster Arterien und kleinster Venen eröffnet. Das was wir mit dem Hautmanometer messen, ist also der höcbste Druck der kleinen Gefässe von dem Kaliber, wie sie in der Haut vorkommen. Wenn deshalb im folgenden der Einfachheit halber von Kapillardruck gesprochen wird, so ist darunter stets der Druck gemeint, unter dem das Blut in den eben erwähnten Gefïssen der Haut steht ${ }^{2}$ ).

\section{Fehler, vor denen man sich hüten muss.}

Die Methode der Bestimmung des Kapillardruckes mit dem Hautmanometer ist auch bei grosser Übung ziemlich umständlich, und

1) Vgl. A. A. Böhm und M. v. David off, Lehrbuch der Histologie des Menschen, 3. Aufl., S. 308. J. F. Bergmann, Wiesbaden 1908. Dort ist auch eine übersichtliche Zeichnung der Blutgefässverteilung nach W. Spalteholz wiedergegeben.

2) Vergleiche dazu das auf S. 347 Gesagte. 
man muss, wenn die Ergebnisse einwandfrei ausfallen sollen, äusserst peinlich verfahren. Es seien deshalb die Punkte zusammengestellt, welche hauptsächlich als Ursache für falsche Bestimmungen in Betracht kommen. Bei dem geringen Bluten der Hautwunden, wie sie für die beschriebenen Versuche gebraucht werden, kann es sich ereignen, dass die Blutung aufgehört hat, noch ehe der Kolben die Höhe erreicht hatte, die dem Druck entspricht. Es ist also notwendig, jedesmal nach dem Versuch nachzusehen, ob auch die Wunde noch blutet. Ein anderer Fehler kann dem Umstande entspringen, dass sich zwischen dem Apparat und der Haut Flüssigkeit entleert. Dann würde natürlich ebenfalls ein zu niedriger Wert bestimmt. Man muss sich also stets davon überzeugen, ob der äussere Rand des Manometers trocken bleibt und, um nicht unnötig Zeit zu verlieren, von Anfang an dafür sorgen, dass der Apparat auch. wirklich dicht aufsitzt. Dafür ist die Hauptbedingung, dass das ganze Manometer vor dem Gebrauch absolut trocken ist, gleichgültig welches der erwähnten Klebemittel man verwendet. Dass aber andererseits der Apparat nach. jedem Versuch gründlich gereinigt werden muss, liegt auf der Hand. Ganz besondere Sorgfalt muss natürlich dem Kolben zugewendet werden. Kommt nur ein Stäubchen zwischen den Kolben und den Zylinder, so bedingt dies ein derartiges Reiben, dass die genaue Einstellung dadurch unmöglich wird. Deshalb ist es am besten, den Kolben gar nicht herauszunehmen. Die Ölschicht, die ihn umgibt, braucht lange Zeit nicht erneuert zu werden. Auch den unteren Teil des Kolbenzylinders, in welchen der Kolben nicht hineinragt, habe ich mit öl gefüllt. Auch dieses Öl soll am besten nicht erneuert werden. Wenn etwas davon verloren ging, wurde es durch einen Tropfen neuen Öles ersetzt; es geschieht dies am besten sofort nach jedem Versuch.

\section{Versuche an der Fingerbeere.}

Die weitaus meisten Versuche wurden an der Volarseite der Fingerspitze ausgeführt. Zum Halten des untersuchten Fingers, gewöhnlich des linken Mittelfingers, schnitt ich in einen grossen Kork eine ungefähr $3 \mathrm{~cm}$ lange und $2 \mathrm{~cm}$ breite Rinne, in welche der Finger gerade hineinpasst. Er wurde mit dem Rücken nach unten hereingelegt, so dass die Fingerbeere gerade horizontal stand.

Nachdem ich an Studenten einige Vorversuche angestellt hatte, ging ich dazu über, in Gemeinschaft mit Herrn Kollegen Kraus 
Untersuchungen über den Druck in den kleinsten Blutgefässen etc. 363

den Blutdruck in den Hautgefässen an einem grösseren Material, nämlich Patienten der Tübinger medizinischen Klinik, zu messen. Bei allen wurde sofort nach der Manometerbestimmung der Kapillardruck mit dem Ochrometer ermittelt und schliesslich nach der Methode von v. Recklinghausen der arterielle Blutdruek auskultatorisch bestimmt. Der Kapillardruck schwankte bei den von uns beobachteten Fällen zwischen 65 und $180 \mathrm{~mm}$ Wasser $=4,7-13,1 \mathrm{~mm}$ Quecksilber. Dabei sind allerdings Fälle, bei denen die Höhe des Kapillardruckes durch krankhafte Prozesse verändert ist, mit einbezogen. Der am meisten vorkommende Wert lag zwischen 100 und $120 \mathrm{~mm}$ Wasser, wenigstens bei solchen Leuten, bei denen die arteriellen Blutdruckverhältnisse als normal zu betrachten waren.

An dieser Stelle seien einige unserer Versuche mitgeteilt.

\section{Versuch rom Mittwoch, 19. November 1913.}

Zimmertemperatur $18^{\circ} \mathrm{C}$. Versuchsperson R., $165 \mathrm{~cm}$ gross, 57 Jahre alt. Klinische Diagnose: Diabetes levis, guter Ernährungszustand.

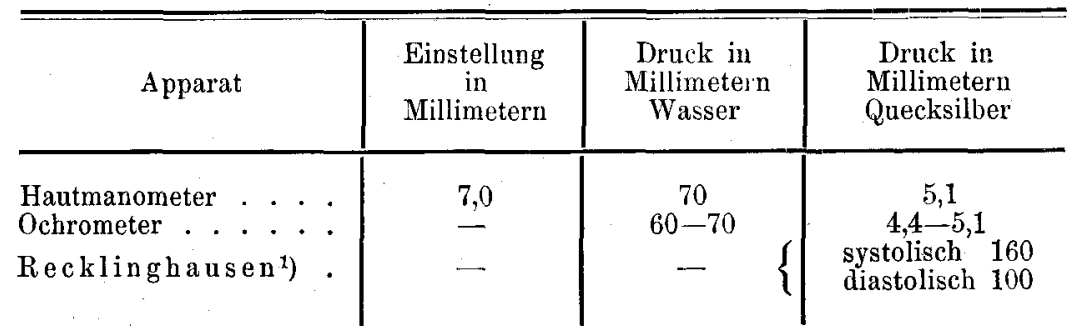

Versuch rom Donnerstag, 20. November 1913.

Zimmertemperatur $17,5^{\circ} \mathrm{C}$. Versuchsperson G., $169 \mathrm{~cm}$ gross, 36 Jahre alt, Klinische Diagnose: Neurasthenie, guter Ernährungszustand.

\begin{tabular}{|c|c|c|c|}
\hline Apparat & $\begin{array}{l}\text { Einstellung } \\
\text { in } \\
\text { Millimetern }\end{array}$ & $\begin{array}{l}\text { Druck in } \\
\text { Millimetern } \\
\text { Wasser }\end{array}$ & $\begin{array}{l}\text { Druck in } \\
\text { Millimetern } \\
\text { Quecksilber }\end{array}$ \\
\hline $\begin{array}{l}\text { Hautmanometer } \\
\text { Ochrometer . } \\
\text { Recklinghausen }\end{array}$ & $\frac{38}{-}$ & $\begin{array}{c}180 \\
170-180 \\
-\end{array}$ & $\begin{array}{cc} & 13,1 \\
& 12,4-13,1 \\
\text { systolisch } & 120 \\
\text { diastolisch } & 96\end{array}$ \\
\hline
\end{tabular}

1) Die Bezeichnung "Recklinghausen" bedeutet die Bestimmung des arteriellen Blutdrucks mit der v. Recklinghausen'schen Arinmanschette. 
Versuch rom Mittwoch, 26. November 1913.

Zimmertemperatur $16^{\circ} \mathrm{C}$. Versuchsperson S., mittelgross, 47 Jahre alt. Klinische Diagnose: Arteriosklerose, Hemiplegie, schlechter Eriährurgszustand.

\begin{tabular}{|c|c|c|c|}
\hline Apparat & $\begin{array}{l}\text { Einstellung } \\
\text { in } \\
\text { Millimetern }\end{array}$ & $\begin{array}{l}\text { Druck in } \\
\text { Millimetern } \\
\text { Wasser }\end{array}$ & $\begin{array}{l}\text { Druck in } \\
\text { Millimetern } \\
\text { Quecksilber }\end{array}$ \\
\hline $\begin{array}{l}\text { Hautmanometer . . . } \\
\text { Oclirometer . . . }\end{array}$ & 16 & $70-80$ & $5,1 \stackrel{5,1}{-5,8}$ \\
\hline Recklinghausen . & - & - & $\begin{array}{ll}\text { systolisch } & 198 \\
\text { diastolisch } & 118\end{array}$ \\
\hline
\end{tabular}

Versuch rom Mittwoch, 26. November 1913.

Zimmertemperatur $16^{\circ} \mathrm{C}$. Versuchsperson B., $169 \mathrm{~cm}$ gross, 49 Jahre alt. Kilinische Diagnose: Arteriosklerose, guter Ernährungszustand.

\begin{tabular}{|c|c|c|c|}
\hline Apparat & $\begin{array}{l}\text { Einstellung } \\
\text { in } \\
\text { Millimetern }\end{array}$ & $\begin{array}{l}\text { Druck in } \\
\text { Nillimetern } \\
\text { Wasser }\end{array}$ & $\begin{array}{l}\text { Druck in } \\
\text { Millimetern } \\
\text { Quecksilber }\end{array}$ \\
\hline $\begin{array}{l}\text { Hautmanometer . . . } \\
\text { Ochrometer. . . . }\end{array}$ & 26,5 & $\begin{array}{c}105 \\
110-120\end{array}$ & $\begin{array}{l}7,7 \\
8,0-8,8\end{array}$ \\
\hline Recklinghausen. & - & - & $\begin{array}{ll}\text { systolisch } & 158 \\
\text { diastolisch } & 100\end{array}$ \\
\hline
\end{tabular}

Versuch vom Donnerstag, 27. November 1913.

Zimmertemperatur $17,5^{\circ} \mathrm{C}$. Versuchsperson H., $163 \mathrm{~cm}$ gross, 29 Jahre alt. Klinische Diagnose: Ulcus duodeni.

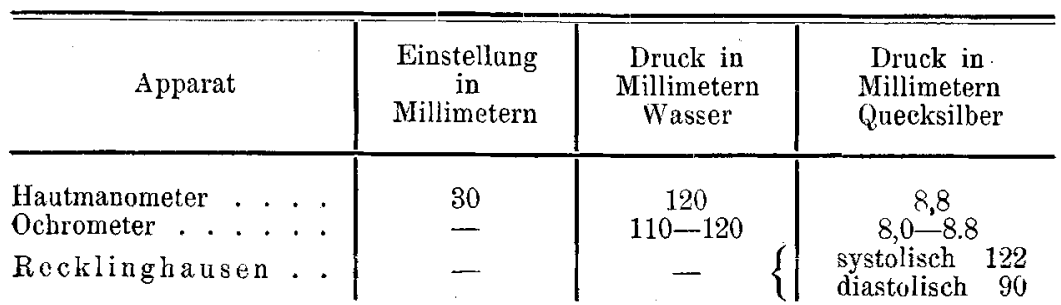

Versuch rom Donnerstag, 27. November 1913.

Zimmertemperatur $17,5^{\circ} \mathrm{C}$. Versuchsperson B., $165 \mathrm{~cm}$ gross, 38 Jahre alt. Klinische Diagnose: Leichtere Herzinsuffizienz, Puls schlecht gefühlt. Fettleibigkeit.

\begin{tabular}{|c|c|c|c|}
\hline Apparat & $\begin{array}{l}\text { Einstellung } \\
\text { in } \\
\text { Millimetern }\end{array}$ & $\begin{array}{l}\text { Druck in } \\
\text { Millimetern } \\
\text { Wasser }\end{array}$ & $\begin{array}{l}\text { Druck in } \\
\text { Millimetern } \\
\text { Quecksilber }\end{array}$ \\
\hline $\begin{array}{l}\text { Hautmanometer . . . } \\
\text { Ochrometer . . . }\end{array}$ & 13 & $\begin{array}{l}65 \\
70\end{array}$ & $\begin{array}{l}4,7 \\
5,1\end{array}$ \\
\hline Recklinghausen. & - & & $\begin{array}{lr}\text { systolisch } & 125 \\
\text { diastolisch } & 98\end{array}$ \\
\hline
\end{tabular}


Untersuchungen über den Druck in den kleinsten Blutgefässen etc. 365

Versuch vom Donnerstag, 4. Dezember 1913.

Zimmertemperatur $17^{\circ} \mathrm{C}$. Versuchsperson P., $165 \mathrm{~cm}$ gross, 61 Jahre alt. Klinische Diagnose: Chronische Nephritis.

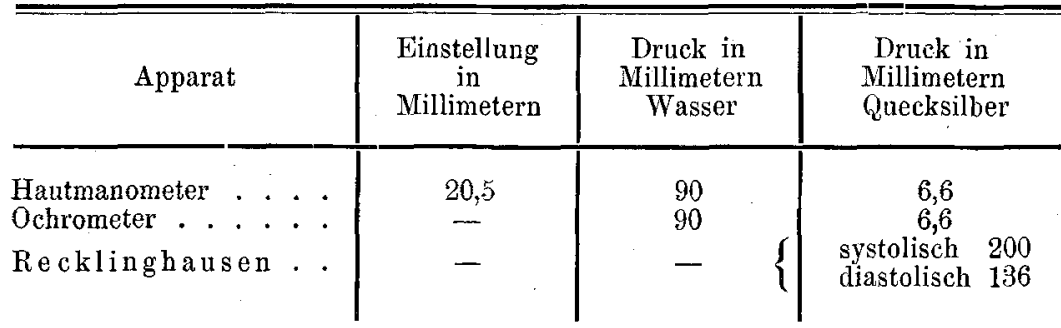

Bei allen diesen Versuchen wurden die Ochrometerbestimmungen so ausgeführt, dass der eine von uns (er sei mit $I$ bezeichnet) in den Tubus sah und die Farbenveränderung beobachtete, wäbrend der andere (II) den Druck über dem Finger langsam erhöbte und an dem Manometer die Höhe des Druckes ablas, sobald der Beobachter I die eben eintretende Farbenveränderung wahrnabm. Auf diese Weise war jede Beeinflussung des Urteils durch eine etwa vorgefasste Meinung ausgeschlossen. Zur Beleuchtung diente eine Gasglühlichtlampe.

Bei der Vergleichung der mit dem Hautmanometer und mit dem Ochrometer gefundenen Ergebnisse zeigte sich eine Übereinstimmung; wie ich sie gar nicht erwartet hatte; denn die Unterschiede betrugen immer nur einige Millimeter Wasser. Ich glaube das als Zeichen dafür ansehen zu dürfen, dass gerade derjenige Farbenunterschierl, den ich gewöhnlich mit dem Ochrometer herstellte, durch die Zusammenpressung der in den oberen Schichten der Cutis enthaltenen Gefässe bedingt ist.

Die zweite Tatsache, welche sich bei den Untersuchungen mit dem Hautmanometer feststellen liess und auf die ich ganz besonders hinweisen möchte, ist die, dass auch hier wieder der sogenannte Kapillardruck sich als recht niedrig erwies.

Schliesslich sei noch die Beziehung des Kapillardruckes" zum arteriellen Blutdruck erwähnt. Bei kachektischen Zuständen, die durch schwere Erkrankungen hervorgerufen waren, zeigte sich bei unseren Versuchen auch der Kapillardruck niedriger. Es ist das eine Tatsache, die auch Landerer ${ }^{1}$ ) bei seinen Kapillardruck-

1) R. Landerer, 1. c. S. 6 des Separatdruckes. 
bestimmungen beobachten konnte. Man darf nun aber nicht schliessen, dass bei einer Erhöhung des arteriellen Blutdruckes unbedingt auch der Kapillardruck grösser sein muss. So fand schon v. Basch ${ }^{1}$ ) in Fällen von Angiosklerose, die sich durch hohen Arteriendruck auszeichneten, den Kapillardruck verhältnismässig niedrig. Den gleichen Zustand konnte er im Tierexperiment durch Vergiftung mit Strychnin hervorrufen ${ }^{2}$ ). Landerer ${ }^{3}$ ) stellte bei Hypertonikern, vorausgesetzt, dass keine venöse Stauung vorlag, häufig einen herabgesetzten Kapillardruck fest.

Auch wir konnten, soweit unsere Beobachtungen reichen, diese Erfahrungen durchaus bestätigen. Bei schlechtem Ernährungszustand betrug der Kapillardruck häufig nur $80 \mathrm{~mm}$ Wasser, in vielen Fällen nur 70, während er bei kräftigen Männern nicht selten eine Höhe von $120-130 \mathrm{~mm}$ erreichte. Bei Hypertonikern lag der Druck in den kleinsten Hautgefässen häufig unter der Norm.

Das, was an unseren Untersuchungen klinisch von Wichtigkeit ist, wird Herr Stabsarzt Dr. Kra uss ${ }^{4}$ ) in einer besonderen Arbeit publizieren.

Versuche am Arme.

Um auch über die Verhältnisse des Kapillardruckes an einer anderen Körperstelle als nur der Fingerspitze ein Urteil zu gewinnen, wurden einige Versuche am Unterarm vorgenommen. Das Manometer wurde statt auf den Finger auf die Dorsalseite des auf dem Tisch ruhenden Armes aufgeklebt, das ist der ganze Unterschied in der Ausführung des Versuches. Der Druck in den Kapillaren der Armhaut war auch derselbe; nur liess sich beobachten, dass der Kolben viel langsamer in die Höhe ging als am Finger; ein Zeichen dafür, dass das Blut spärlicher aus der Wunde heraustrat. Dieser Unterschied ist ganz verständlich, wenn man beilenkt, dass die Haut der Fingerbeeren viel reicher an Blutgefässen ist als die Armbaut. Deshalb werden an den Fingern mehr Kapillaren angeschnitten als bei gleich grosser Verletzung am Arm. Liegen aber 100 der kleinsten

1) S. v. Basch, Über die Messing des Kapillardruckes am Menschen und deren physiologische und klinische Bedeutung. Wiener klin. Rundschau Bd. 14 S. 549 (574). 1900 .

2) S. v. Basch, Experimentelle und klinische Untersuchungen übér den Kapillardruck. Intern. Beitr. zur inn. Med. Bd. 1 S. 65 (72). 1912.

3) R. L anderer, 1. c. Separatdruck S. 7.

4) Die Arbeit von Krauss wird demnächst in den Volkmann'schen klinischen Heften erscheinen. 
Blutgefässe in der Schnittwunde, dann kommt natürlich in der Zeiteinheit mehr Blut heraus, als wenn man vielleicht nur 10 derselben eröffnet.

Mit dem verhältnismässig geringen Blutreichtum der Armhaut hängt es wohl auch zusammen, dass man häufig sogar nach wiederholtem Einstich gar keine Blutung bekommt, so dass der Versuch nicht ausgeführt werden kann. Dies ist der Grund, weshalb die überwiegende Mehrzahl meiner Versuche an der Fingerbeere vorgenommen wurde.

Wenn sich bei meinen Beobachtungen in den kleinsten Gefässen nur ein Druck von $80-100 \mathrm{~mm}$ Wasser feststellen liess, so muss man natürlich berücksichtigen, dass alle meine Versuche an der Hand, während sie sich nur wenig unter der Herzhöhe befand, vorgenommen wurden. Es soll selbstverständlich nicht bestritten werden, dass an Körperteilen, die weiter unterbalb des Herzens liegen, der Kapillardruck ein höherer ist. So beträgt z. B. beim stehenden Menschen sogar schon der Venendruck im Fuss über $1200 \mathrm{~mm}$ Wasser. Doch war es mir bisher noch nicht möglich, den Kapillardruck der unteren Extremitäten zu bestimmen.

\section{Zur Kritik des Apparates.}

Wie aus dem Mitgeteilten hervorgeht, wurden mit dem Hautmanometer Werte für den Kapillardruck gefunden, die wesentlich kleiner sind, als nach den Ergebnissen der meisten früheren Untersuchungen zu erwarten war. Deshalb muss die Frage aufgeworfen werden, ob der ermittelte Druck nicht durch Fehler, die dem Apparat anhaften, zu klein ausfällt. In erster Linie ist zu bedenken, dass das Manometer mit seinem eigenen Gewicht, das etwa $10 \mathrm{~g}$ beträgt, dem Finger aufsitzt. Dadurch wird eine ringförmige Zone der Haut gepresst. Es liesse sich annehmen, dass infolge davon die zuführenden Arterien verengert werden, so dass in dem eingeschlossenen Gebiet, in dem ja die Bestimmung ausgeführt wird, der Kapillardruck sinkt. Dass diese Erwägung der Wirklichkeit nicht entspricht, davon kann man sich durch Beobachtung der Hautfarbe überzeugen. Setzt man den Ring $a$ (Fig. 3 S. 353) auf die Fingerbeere, dann wird das eingeschlossene Hautstück nicht blässer, wie es bei teilweisem Verschluss der zuführenden Gefässe sein müsste; es tritt selbst dann keine Farbenveränderung ein, wenn man zum Aufdrücken eine viel grössere Kraft anwendet, als dem Gewicht 
des Apparates entspricht. Wenn durch das Aufsetzen des Apparates wirklich eine Beeinflussung des Druckes in den Hautgefässen stattfindet, dann ist viel eher anzunehmen, dass infolge einer venösen Staunng der Druck unverhältnismässig gross ist. Denn die Venen werden naturgemäss eher komprimiert als die Arterien. Der zweite Finwand, den man gegen die Methode machen kann, ist der, dass der Einstich vielleicht zu tief gemacht wurde, wodurch nicht nur die allerkleinsten Gefässe der Haut verletzt wurden. Aber auch in diesem Falle würde der gefundene Druck eher zu gross als zu klein ausfallen. Wenn man demnach die zwei angeführten Bedenken als zu Recht bestehend anerkennen wollte, dann müsste man sagen: Der Kapillardruck ist noch kleiner, als er in den beschriebenen Versuchen gefunden wurde.

Hat die Erwärmung der in dem Apparat enthaltenen Flïssigkeit einen Einfluss auf die Einstellung des Kolbens?

Wird das Manometer mit einer Flüssigkeit gefüllt, welche etwas kälter ist als die Hauttemperatur, dann kann möglicherweise im Verlauf des Versuches eino Erwärmung der Flüssigkeit eintreten. Man muss also daran denken, dass der Kolben schon durch die Ausdehnung infolge von Erwärmung etwas gehoben wird, so dass die fur den Kapillardruck gefundenen Werte zu gross ausfallen. Ein anderes Mal könnte die vor dem Versuch erwärmte Lösung sich in dem Manometer abkühlen; ihr Volumen würde kleiner und dadurch die Bestimmungen zu niedrig.

Die Menge der im Apparat enthaltenen Flüssigkeit, welche ihre Temperatur verändern könnte, lässt sich leicht berechnen. Der Durchmesser der unteren Öffuung des Apparates beträgt $8,0 \mathrm{~mm}$, also der Radius $4 \mathrm{~mm}$. Die Höhe des Hohlraumes, der zwischen der Haut und dem Zapfen $e$ (Fig. 2 S. 352) übrig bleibt, beträgt $5 \mathrm{~mm}$. Demnach misst die Bodentläche $4^{2} \pi=16 \cdot 3,14=50,24 \mathrm{qmm}$ und der Inhalt des Hohlraumes $5 \cdot 50,24=251,2 \mathrm{cbmm}$. Dazu kommt noch der Inhalt des Ansatzrohres $b$ (Fig. 2 S. 350). Den Durchmesser seiner Lichtweite nehme ich zu $1 \mathrm{~mm}$ an (absichtlich etwas zu gross), bis zur oberen Ausflussöffnung ist es $25 \mathrm{~mm}$ lang. Sein Inhalt beläuft sich dann auf $0,5^{2} \pi \cdot 25=19,6 \mathrm{cbmm}$. Also beträgt die in dem Manometer enthaltene Flüssigkeitsmenge $251,2+19,6=270,8 \mathrm{cbmm}$ oder abgerundet $270 \mathrm{cbmm}$.

Um für die Entstehung eines Fehlers möglichst günstige Ver- 
hältnisse zu bieten, nehme ich den ganz extremen Fall an, dass der Apparat mit Kochsalzlösung von $20^{\circ} \mathrm{C}$. gefüllt wird, und dass die Flüssigkeit sich während des Versuchs auf $30^{\circ} \mathrm{C}$. erwärmt, dass also eine Temperaturzunahme um $10^{\circ} \mathrm{C}$. stattfindet. Nun wissen wir aber, dass 1 cbmm Wasser, dessen Ausdehnung sich wenig von derjenigen der physiologischen Kochsalzlösung unterscheidet, bei einer Erwärmung von $20^{\circ}$ auf $30^{\circ}$ C. sich um nicht ganz $0,002509 \mathrm{cbmm}$ ausdehnt ${ }^{1}$ ). Die in dem Apparat enthaltenen $270 \mathrm{cbmm}$ nehmen demnach bei Erwärmung von 20 auf $30^{\circ} \mathrm{C}$. um $270 \cdot 0,002509 \mathrm{cbmm}=$ $0,67743 \mathrm{cbmm} z u$. Eine solche Volumenzunahme bedingt aber nach dem auf S. 355 Gesagten nur eine Erhebung des Kolbens um $\frac{0,68}{3,14}=0,21 \mathrm{~mm}$.

$\mathrm{Ob}$ die Veränderung der Temperatur eine Verschiebung des Kolbens bedingt, wurde auch experimentell geprüft. Dazu wurde der Apparat in der gewöhnlichen Art aufgeklebt, gefüllt und der Teil mit dem Kolben aufgesetzt, alles wie bei der richtigen Bestimmung, bloss wurde die Haut nicht angestochen. Bei allen diesen Versuchen, die in grosser Zahl vorgenommen wurden, bewegte sich der Kolben keine Spur. Wir haben also damit auch den experimentellen Nachweis, dass die Einstellung des Kolbens dureh Temperaturänderungen in keiner Weise beeinflusst wird.

\section{Kurze Zusammenstellung der gewonnenen Ergebnisse.}

1. Der mit dem Ochrometer bestimmte Druck in den Hautgefässen der Fingerbeere betrug bei mehreren Versuchspersonen $90-120 \mathrm{~mm}$ Wasser $=6,6-8,8 \mathrm{~mm}$ Quecksilber.

2. Bei einer grösseren Zahl von Patienten der medizinischen Klinik, die ebenfalls mit dem Ochrometer untersucht wurden, schwankte der Druck zwischen 65 und $180 \mathrm{~mm}$ Wasser. Darunter befanden sich auch Leute, deren Blutdruckverhältnisse durch pathologische Prozesse erheblich verändert waren.

3. Gleichzeitig mit dem Ochrometer wurden die Patienten auch mit dem Hautmanometer untersucht, einem Apparat, der es gestattet, den Druck zu messen, unter dem das Blut aus einer kleinen Verletzung der Fingerhaut ausströmt. Dabei ergab sich, dass die mit

1) Müller-Pouillets Lehrb. d. Physik, 9. Aufl., Bd. 2 Abt. 2 S. 92. Vieweg \& Sohn, Braunschweig 1898. 
dem Ochrometer und die mit dem Hautmanometer gefundenen Werte sebr gut miteinander übereinstimmten.

4. Mit dem Hautmanometer am Arm angestellte Versuche führten zu den gleichen Ergebnissen wie die am Finger vorgenommenen.

5. Aus den bis jetzt angestellten Untersuchungel ergab sich bei normalem arteriellem Blutdruck in den kleinen Gefässen der Fingerhaut ein Druck von $90-130 \mathrm{~mm}$ Wasser $=6,6-9,5 \mathrm{~mm}$ Quecksilber.

6. Bei kachektischen Zuständen war der Kapillardruck niedriger, als es der Norm entspricht, bis herunter zu $70 \mathrm{~mm}$ Wasser. Das Gleiche liess sich beobachten in manchen Fällen von abnorm erhöhtem arteriellem Blutdruck, z. B. bei Schrumpfniere und bei Arteriosklerose.

Zum Schlusse sei es mir gestattet, Herrn Professor 0. Müller für die freundliche Überlassung des klinischen Materials meinen herzlichsten Dank auszusprechen. 